\title{
Drug resistant tuberculosis in prisons in Azerbaijan: case study
}

\author{
R Coninx, G E Pfyffer, C Mathieu, D Savina, M Debacker, F Jafarov, I Jabrailov, A Ismailov, F Mirzoev, \\ $\mathrm{R}$ de Haller, F Portaels
}

\begin{abstract}
Objectives: To document the existence of drug resistance in a tuberculosis treatment programme that adheres strictly to the DOTS principles (directly observed treatment, short course) and to determine the extent of drug resistance in a prison setting in one of the republics of the former Soviet Union.

Design: Case study.

Setting: Central Penitentiary Hospital in Baku, the referral centre for tuberculosis patients from all prisons in Azerbaijan.

Subjects: Prisoners with tuberculosis: 28 selected patients not responding clinically or bacteriologically to the standard treatment (group 1) and 38

consecutive patients at admission to the programme (group 2).

Main outcome measures: Drug resistance of

Mycobacterium tuberculosis strains grown from sputum. Results: All the non-responding patients (group 1) had strains resistant to at least one drug. $25(89 \%)$ of the non-responding patients and nine (24\%) of the consecutive patients had $M$ tuberculosis strains resistant to both rifampicin and isoniazid. A further 17 patients in group 2 had strains resistant to one or more first line drugs.

Conclusions: Drug resistant $M$ tuberculosis strains are common in prisons in Azerbaijan. Tuberculosis problems tend to be worse in prisons, but prisoners and former prisoners may have an important role in the transmission of tuberculosis, particularly of drug resistant forms, in the community. National programmes to control tuberculosis will have to take into account and address the problems in prisons to ensure their success.
\end{abstract}

\section{Introduction}

Tuberculosis is an increasing problem in Azerbaijan. ${ }^{12}$ In prisons this problem is compounded by overcrowding, poor general health, high prevalence of risk groups, late case finding, and incomplete treatments. As a result prevalences in prisons in Azerbaijan were almost 50 times higher than the country's average and the mortality was as high as $24 \%$ in $1994 .^{3}$ The common practice of self treatment with drugs provided by family members often results in treatment at insufficient doses and with multiple interruptions. The incidence of HIV infection in the general population is low, ${ }^{4}$ and there is no evidence that HIV plays an important part in the current epidemic of tuberculosis in prisons. Drug resistance is known to exist in the countries of the former Soviet Union, but exact data have rarely been presented. ${ }^{5}{ }^{6}$ No data are available for Azerbaijan.

The International Committee of the Red Cross has implemented a tuberculosis control programme in the
Central Penitentiary Hospital in Baku, Azerbaijan, which is the country's only treatment centre for patients with tuberculosis in prison. The programme started in June 1995 and used a short course of directly observed treatment (DOTS) as recommended by the World Health Organisation ${ }^{7}$ and the International Union Against Tuberculosis and Lung Diseases. ${ }^{8}$ The objective of this study was to document the existence and the extent of multidrug resistance.

\section{Subjects and methods}

We analysed sputum from two groups of patients in whom Mycobacterium tuberculosis was identified. Group 1 comprised 28 patients who did not respond, either clinically or bacteriologically, after a minimum of eight weeks to the fully supervised WHO treatment regimen. Group 2 comprised 38 patients consecutively enrolled over four weeks from whom sputum was taken before the start of the treatment. The two groups were mutually exclusive. One sputum sample was taken from each patient. We supervised collection of sputum to avoid irregularities.

\section{Treatment protocols}

We included only patients whose sentence was long enough to allow a full course of treatment. Patients received the WHO recommended DOTS regimen in the recommended doses. ${ }^{9}$ For new cases, defined as having never been treated or treated for less than a month, treatment comprised daily doses of rifampicin, isoniazid, pyrazinamide, and streptomycin for two months followed by rifampicin and isoniazid thrice weekly for four months. For failure and relapse cases initial treatment was daily doses of rifampicin, isoniazid, pyrazinamide, and ethambutol for three months plus streptomycin for the first two months followed by rifampicin, isoniazid, and ethambutol thrice weekly for five months. Failure of treatment was defined as patients who had stopped treatment after having taken drugs for at least one month. Relapse cases were patients who had previously completed a full treatment course. Medicines were bought from the International Dispensary Association in Amsterdam and imported to ensure compliance with international standards. Drug intake was directly supervised by our staff, who observed the patients swallowing the medicines. This allowed us to achieve a compliance rate of $94 \%$.

\footnotetext{
Microbiological analyses

Microscopy-Smears were stained with the ZiehlNeelsen technique. ${ }^{10}$

Culture-Sputum specimens were collected in the morning, stored at $4^{\circ} \mathrm{C}$, and taken in batches to the laboratories for processing. They were decontaminated by the Petroff method or by $N$-acetyl-L-cysteine and
}

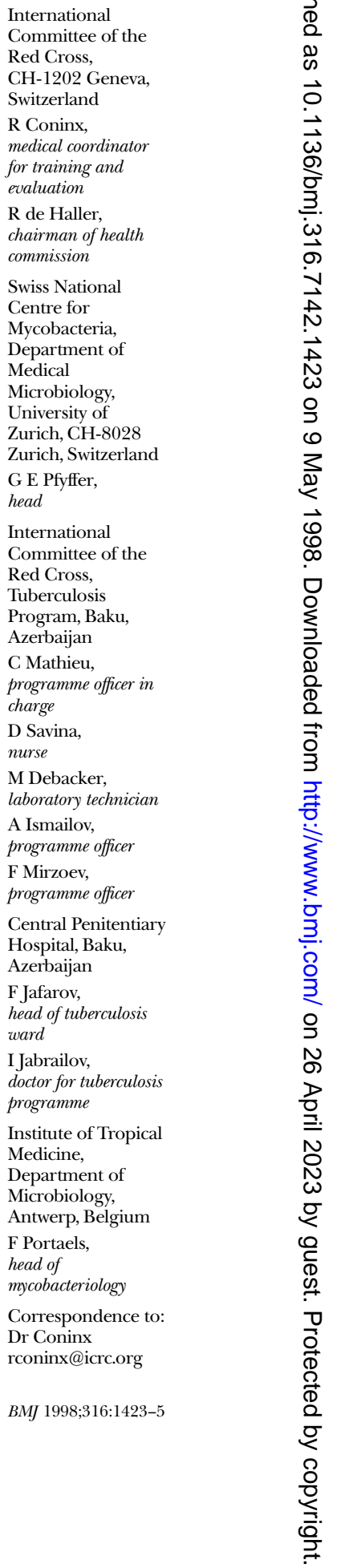


sodium hydroxide ${ }^{10}$ inoculated on to solid media, and incubated at $36^{\circ} \mathrm{C}$ for eight weeks in $6 \%$ carbon dioxide. In addition, $0.5 \mathrm{ml}$ of the sediment was cultivated by the radiometric BACTEC 460 TB technique. $^{11}$

Identification of $M$ tuberculosis-Routine biochemical methods ${ }^{10}$ and Accuprobe culture confirmation kits (Gen-Probe, San Diego, CA, USA) were used to identify the isolates.

Susceptibility testing-Susceptibility to first line drugs (isoniazid, rifampicin, ethambutol, and pyrazinamide) and to streptomycin was tested with the radiometric BACTEC 460 TB method (isoniazid $0.2 \mu \mathrm{g} / \mathrm{ml}$; rifampicin $2.0 \mu \mathrm{g} / \mathrm{ml}$; ethambutol $2.5 \mu \mathrm{g} / \mathrm{ml}$; pyrazinamide $100 \mu \mathrm{g} / \mathrm{ml}$; streptomycin $2 \mu \mathrm{g} / \mathrm{ml}$ ) as well as by the proportion method on solid medium. Resistance was defined according standard protocols. ${ }^{12}$

\section{Results}

$M$ tuberculosis was isolated from all sputum specimens $(n=66)$. The table shows the antibiotic resistance pattern of the strains. In group 1, 25 of the strains $(89 \%)$ proved to be multidrug resistant (resistant to rifampicin and isoniazid). None was fully susceptible. The strains of all new cases and 14 out of 17 failure or relapse cases $(82 \%)$ were multidrug resistant.

In group 2, nine strains (24\%) were multidrug resistant and only $12(32 \%)$ were fully susceptible. Three (15\%) strains in the 20 new cases and six (33\%) strains in the 18 failure and relapse cases were multidrug resistant.

\section{Discussion}

Our data show that drug resistance is common in the Central Penitentiary Hospital in Baku and an important factor in non-response to treatment.

Susceptibility patterns of $M$ tuberculosis strains among non-responding cases (group 1) and consecutive cases (group 2)

\begin{tabular}{|c|c|c|c|c|c|}
\hline \multirow{2}{*}{$\begin{array}{l}\text { Total No of cases } \\
\text { (new cases) }\end{array}$} & \multicolumn{5}{|c|}{ Drug resistance } \\
\hline & Isoniazid & Rifampicin & Ethambutol & Pyrazinamide & Streptomycin \\
\hline \multicolumn{6}{|l|}{ Group 1 (n=28) } \\
\hline $8(2)$ & $R$ & $R$ & $\mathrm{R}$ & $\mathrm{R}$ & $\mathrm{R}$ \\
\hline $4(1)$ & $R$ & $\mathrm{R}$ & $\mathrm{R}$ & NA & $\mathrm{R}$ \\
\hline 4 & $R$ & $\mathrm{R}$ & $\mathrm{R}$ & $S$ & $\mathrm{R}$ \\
\hline $6(6)$ & $R$ & $R$ & $S$ & $\mathrm{R}$ & $\mathrm{R}$ \\
\hline $2(1)$ & $\mathrm{R}$ & $\mathrm{R}$ & $\mathrm{S}$ & $\mathrm{S}$ & $\mathrm{R}$ \\
\hline 1 & $R$ & $S$ & $\mathrm{~S}$ & $\mathrm{R}$ & $\mathrm{R}$ \\
\hline 2 & $\mathrm{R}$ & $S$ & $S$ & $\mathrm{R}$ & $S$ \\
\hline $1(1)$ & $\mathrm{R}$ & $\mathrm{R}$ & $\mathrm{S}$ & $\mathrm{R}$ & $S$ \\
\hline No (\%) resistant & $28(100)$ & $25(89)$ & $16(57)$ & $18(75)^{*}$ & $25(89)$ \\
\hline \multicolumn{6}{|l|}{ Group $2(n=38)$} \\
\hline $2(1)$ & $R$ & $R$ & $\mathrm{R}$ & $\mathrm{R}$ & $\mathrm{R}$ \\
\hline 1 & $R$ & $R$ & $\mathrm{R}$ & NA & $\mathrm{R}$ \\
\hline $3(1)$ & $R$ & $R$ & $\mathrm{R}$ & $\mathrm{S}$ & $\mathrm{R}$ \\
\hline $3(1)$ & $\mathrm{R}$ & $R$ & $\mathrm{~S}$ & $\mathrm{~S}$ & $\mathrm{R}$ \\
\hline 1 & $\mathrm{R}$ & $S$ & $\mathrm{R}$ & $\mathrm{R}$ & $\mathrm{R}$ \\
\hline $11(5)$ & $R$ & $S$ & $\mathrm{~S}$ & $S$ & $\mathrm{R}$ \\
\hline $1(1)$ & $\mathrm{R}$ & $S$ & $S$ & $\mathrm{R}$ & $S$ \\
\hline 1 & $R$ & $S$ & $\mathrm{~S}$ & $S$ & $\mathrm{~S}$ \\
\hline $3(3)$ & $S$ & $S$ & $\mathrm{~S}$ & $S$ & $\mathrm{R}$ \\
\hline $1(1)$ & $S$ & $S$ & $S$ & NA & $\mathrm{S}$ \\
\hline $11(7)$ & $S$ & $S$ & $S$ & $\mathrm{~S}$ & $\mathrm{~S}$ \\
\hline No (\%) resistant & $23(60)$ & $9(24)$ & $7(18)$ & $4(11) \dagger$ & $24(63)$ \\
\hline
\end{tabular}

$R=$ resistant, $S=$ susceptible, NA=not available. ${ }^{*} 18$ out of 24 . $† 4$ out of 36 .
- Tuberculosis is an important problem in prisons in Azerbaijan

- Multidrug resistant tuberculosis was common and an important cause of non-response to standard treatment

- National tuberculosis control programmes must include prisons and take account of drug resistance

- Unless WHO recommended treatment protocols are followed the problem of multidrug resistant tuberculosis may result in untreatable tuberculosis which will spread to the general community

Although these data cannot be generalised because of the limited number of samples investigated, they have implications for the management of tuberculosis in prisons. Treatment practices in Azerbaijan need to be reviewed as frequent monotherapy and multiple interrupted treatment courses undoubtedly contribute to the widespread multidrug resistance problem.

Our results suggest that the new cases were not really new or that patients became infected with drug resistant strains. We used the WHO definition of new cases, and many patients had taken antituberculous drugs for up to four weeks. It may be more appropriate to restrict the definition of new cases to patients without any history of treatment for tuberculosis.

The epidemiological implications of tuberculosis in prisons in the republics of the former Soviet Union may be more serious than commonly assumed. For example, data from Mariinsk (Siberia) indicate that a third of patients with tuberculosis have been in prison. ${ }^{13}$ A similar trend is seen in Ivanovo, Russia (A Khomenko, personal communication). These data suggest that prisoners and former prisoners may in future have an important role in the transmission of tuberculosis, particularly of multidrug resistant forms, in the community.

National tuberculosis programmes will have to take into account the problems currently existing in prisons. The extent of tuberculosis in prisons, which is much greater than in the community, is underreported. Statistics on tuberculosis in the republics of the former Soviet Union are provided by the Ministry of Health and do not include data from prisons, which are under the jurisdiction of the Ministry of Justice (or in some countries the Ministry of Interior). Organisations running tuberculosis control programmes in prisons need to determine the presence and extent of multidrug resistance before starting a project.

We thank the staff of Baku prison and the Ministry of Justice in Azerbaijan, whose efforts to tackle the problem of tuberculosis in prisons led to the establishment of the current DOTS programme and who continue to provide all necessary support.

Contributors: RC initiated and coordinated the project, participated in discussion and analysis of the data, wrote the paper, and is the guarantor. GEP guided the laboratory analyses, participated in the discussion and analysis of the data and helped write and revise the paper. CM participated in the design of the project, was in charge of the patient management and sputum collection, and participated in discussion and analysis of the data and writing the paper. DS participated in the patient 
management, sputum collection, and discussions. MD performed the laboratory examinations in Baku, prepared the sputum specimens, and participated in discussion and analysis of the data. FJ was in charge of patient management, IJ participated in the patient management. AI helped with laboratory examinations and sputum collection. FM helped with patient management and data collection. $\mathrm{RdeH}$ initiated the project and participated in the discussion and analysis of the data and in writing the paper. FP participated in the analysis of specimens, discussion and analysis of the data, and writing the paper.

Funding: The Damien Foundation (Brussels) partly funded the laboratory investigations in Antwerp and the Zurich Lung Association those in Zurich.

Conflict of interest: None.

1 World Health Organisation. Tuberculosis trends in central and eastern Europe and countries of the former USSR. Wkly Epidemiol Rec 1995;4:21-4.

2 Raviglione MC, Rieder HL, Styblo K, Khomenko AG, Esteves K, Kochi A. Tuberculosis trends in eastern Europe and the former USSR. Tubercle Lung Dis 1994;75:400-6.

3 Coninx R, Eshaya-Chauvin B, Reyes H. Tuberculosis in prisons. Lancet $1995 ; 346: 1238-9$.

4 European Centre for the Epidemiological Monitoring of AIDS HIV/AIDS surveillance in Europe. Quarterly report (No 52). Saint Maurice, France: ECEMAIDS, 1996:11.
5 Khomenko AG. The course and outcome of pulmonary tuberculosis due to drug resistant mycobacteria. 17th Annual meeting, European Society for Mycobacteriology. Paris: Institut Pasteur, 1996.

6 Drobniewski F, Taylor E, Ignatenko N, Paul J, Conolly M, Nye P, et al. Tuberculosis in Siberia. 1. An epidemiological and microbiological assessment. Tubercle Lung Dis 1996;77:199-206.

7 World Health Organisation. WHO report on the tuberculosis epidemic, 1995. Stop TB at the source. Geneva: WHO, 1995. (WHO/TB/95.183.)

8 International Union Against Tuberculosis and Lung Disease. Tuberculosis guide for low income countries. 4th ed. Paris : IUATLD, 1996.

9 World Health Organisation. Treatment of tuberculosis. Guidelines for national programmes. Geneva: WHO, 1993. (WHO/TB.91.161.)

10 Kent PT, Kubica G. Public health mycobacteriology. A guide for the level IIt laboratory. Atlanta: US Department of Health and Human Services, Centers for Disease Control, 1985

11 Morgan MA, Horstmeier CD, De Young DR, Roberts GD. Comparison of a radiometric method (BACTEC) and conventional culture media for recovery of mycobacteria from smear-negative specimens. J Clin Microbiol 1983;18:384-8.

12 Inderlied CB, Nash K. Antimycobacterial agents: in vitro susceptibility testing, spectra of activity, mechanisms of action and resistance, and assays for activity in biologic fluids. In: Lorian V, ed. Antibiotics in laboratory medicine. 4th ed. Baltimore: Williams and Wilkins, 1996:127-75.

13 Kluge H. TB programme in Siberia. Like a scene from an old movie. Contact 1997;45:24.

(Accepted 5 February 1998)

\title{
Prospective, hospital based study of fever in children in the United Kingdom who had recently spent time in the tropics
}

\author{
John L Klein, Guy C Millman
}

Published data are lacking on the subject of imported infections in children. As general practitioners and paediatricians in the United Kingdom are frequently involved in the assessment of children with such infections, this lack of information may hinder optimal management. We report the results of a one year prospective, hospital based study of all children with fever admitted to our paediatric ward who had recently spent time in the tropics.

\section{Methods, subjects, and results}

From August 1996 to July 1997 all children aged 16 years and under who were admitted with a fever (oral temperature $>37.5^{\circ} \mathrm{C}$ ) and had been in a tropical country within the previous four weeks were entered into the study; details of the few children who had a fever and had been in the tropics but were managed as outpatients were not recorded. Demographic, clinical, and laboratory features were recorded on a standard proforma.

In all, 31 children (18 boys) met the entry criteria; the median age was 4 years (range 5 months to 15 years). The regions visited were south Asia (19), sub-Saharan Africa (11), and the Caribbean (1). Twenty one children were normally resident in the United Kingdom, five in Africa, and five in south Asia; 23 were of south Asian ethnic origin, and eight were Afro-Caribbean. Of the 20 children normally resident in the United Kingdom who had visited a malarious region, only three had been fully compliant with an accepted regimen of antimalarial prophylaxis ${ }^{1}$; eight had taken no prophylaxis, and the other nine were poorly compliant, especially with proguanil.
The table shows the primary diagnoses at discharge from hospital. Fourteen children had non-specific, self limiting illnesses of presumed viral origin. Of the remaining 17 children, seven had potentially fatal infections requiring rapid diagnosis and antimicrobial treatment. All three cases of falciparum malaria were acquired in sub-Saharan Africa, and the single case of vivax malaria originated from India. Ten children had notifiable infectious diseases, and there were no deaths.

Primary diagnoses at discharge in 31 children admitted to hospital with fever after arriving in United Kingdom from the tropics

\begin{tabular}{lccc} 
& \multicolumn{3}{c}{ No of cases } \\
\cline { 2 - 4 } Diagnosis & Resident in UK & $\begin{array}{c}\text { Not resident } \\
\text { in UK }\end{array}$ & Total \\
\hline Non-specific fever & 10 & 4 & 14 \\
\hline Malaria*: & 2 & 1 & 3 \\
\hline Falciparum malaria & 0 & 1 & 1 \\
\hline Vivax malaria & 3 & 0 & 3 \\
\hline Bacillary dysentery ${ }^{*} \dagger$ & 2 & 0 & 2 \\
\hline Dengue fever & 2 & 0 & 2 \\
\hline Typhoid ${ }^{*}$ & 0 & 1 & 1 \\
\hline Acute hepatitis A & 1 & 0 & 1 \\
\hline Bacterial lymphadenitis & 0 & 1 & 1 \\
\hline Pneumonia & 0 & 1 & 1 \\
\hline Pneumocystis carinii pneumoniał & 0 & 1 & 1 \\
\hline Acute myeloid leukaemia & 1 & 0 & 1 \\
\hline Streptococcal throat infection & & &
\end{tabular}

*Notifiable infections.

†Positive stool isolates: shigella (1 case), salmonella (1).

‡Newly diagnosed HIV infection.
Children's Services Directorate, Northwick Park and St Mark's NHS Trust, Harrow, Middlesex HA1 3UJ John L Klein, senior house officer Guy C Millman, senior house officer Correspondence to: Dr J L Klein, 177 Purves Road, Kensal Rise, London NW10 5TH Johnlklein@email. msn.com

BMJ 1998;316:1425-6 